\title{
MOMENTS OF MEASURE ORTHOGONALIZING THE 2-DIMENSIONAL CHEBYSHEV POLYNOMIALS
}

\author{
ŁUKASZ JAN WOJAKOWSKI \\ Mathematical Institute, University of Wrockaw \\ Pl. Grunwaldzki 2/4, 50-384 Wroctaw, Poland \\ E-mail: Lukasz.Wojakowski@math.uni.wroc.pl
}

\begin{abstract}
We calculate the moments $m_{k, 0}$ of the measure orthogonalizing the 2-dimensional Chebyshev polynomials introduced by Koornwinder.
\end{abstract}

In $[\mathrm{K}]$ Koornwinder introduced a two-dimensional analogue of the classical Chebyshev polynomials of the second kind. They are defined by the following recurrence relations:

$$
\begin{gathered}
P_{-1, l}(z, \bar{z})=0, \quad P_{k,-1}(z, \bar{z})=0 \\
P_{0,0}(z, \bar{z})=1, \\
P_{1,0}(z, \bar{z})=z, \quad P_{0,1}(z, \bar{z})=\bar{z}, \\
z P_{k, l}(z, \bar{z})=P_{k+1, l}(z, \bar{z})+P_{k-1, l+1}(z, \bar{z})+P_{k, l-1}(z, \bar{z}), \\
\bar{z} P_{k, l}(z, \bar{z})=P_{k, l+1}(z, \bar{z})+P_{k+1, l-1}(z, \bar{z})+P_{k-1, l}(z, \bar{z})
\end{gathered}
$$

The total degree of $P_{k, l}(z, \bar{z})$ is thus $k+l$. For general properties of multidimensional orthogonal polynomials see for instance [DX].

Those polynomials form a system orthonormal with respect to the weight function

$$
\mu(z, \bar{z})=\frac{1}{2 \pi^{2}} \sqrt{-z^{2} \bar{z}^{2}+4 z^{3}-4 \bar{z}^{3}+18 z \bar{z}-27}
$$

over the region $S$ inside the Steiner's hypocycloid $\partial S$ :

$$
\partial S(\theta)=2 e^{i \theta}+e^{-2 i \theta}, \quad 0 \leq \theta<2 \pi .
$$

2000 Mathematics Subject Classification: 60E99.

Key words and phrases: moments, Chebyshev polynomials, multidimensional polynomials.

Partially sponsored with KBN grant no 2P03A00723 and RTN HPRN-CT-2002-00279.

The author wishes to thank Philippe Biane for help and discussion.

The paper is in final form and no version of it will be published elsewhere. 
that is, satisfy the following relation:

$$
\iint_{S} P_{m, n}(z, \bar{z}) P_{k, l}(z, \bar{z}) \mu(z, \bar{z}) d x d y=\delta_{m, k} \delta_{n, l} .
$$

We are concerned with the problem of calculating the moments $m_{k, l}$ of the measure $\mu(z, \bar{z}) d x d y$ :

$$
m_{k, l}=\iint_{S} z^{k} \bar{z}^{l} \mu(z, \bar{z}) d x d y .
$$

We limit ourselves to the specific case of $m_{k, 0}$ :

$$
m_{k, 0}=\iint_{S} z^{k} \mu(z, \bar{z}) d x d y
$$

Then, using the recurrence relations, we can write (omitting for simplicity the explicit dependence of $P_{k, l}$ on $\left.(z, \bar{z})\right)$ :

$$
\begin{aligned}
z^{k} & =z^{k-1}\left(z P_{0,0}\right) \\
& =z^{k-1} P_{1,0}=z^{k-2}\left(z P_{1,0}\right) \\
& =z^{k-2}\left(P_{2,0}+P_{0,1}\right)=z^{k-3}\left(z P_{2,0}+z P_{0,1}\right) \\
& =z^{k-3}\left(P_{3,0}+P_{1,1}+P_{1,1}+P_{0,0}\right) \\
& =\ldots=z^{0} \sum_{i} P_{u_{i}(k), v_{i}(k)}=\sum_{i} P_{u_{i}(k), v_{i}(k)} .
\end{aligned}
$$

We see that every multiplication by $z$ of the initial $z^{k}$ can be eliminated by application of the formula (1). Each use of this formula replaces every orthonormal polynomial $P_{u, v}$ by the respective sum of three others (or fewer, should any of the indices go negative). Eventually, when all multiplications by $z$ are exhausted, we are left with a sum of orthonormal polynomials $P_{u_{i}(k), v_{i}(k)}$, where $i$ runs from 1 to the number of polynomials with nonnegative indices and $k$ means that $k$ applications of formula (1) have been made. In order to calculate the moments $m_{k, 0}$ we have now

$$
m_{k, 0}=\iint_{S} \sum_{i} P_{u_{i}(k), v_{i}(k)} \mu d x d y=\sum_{i} \iint_{S} P_{0,0} P_{u_{i}(k), v_{i}(k)} \mu d x d y .
$$

Because of the orthogonality relations we see now that the moment is equal to the number of pairs of indices $u_{i}(k), v_{i}(k)$ such that $u_{i}(k)=v_{i}(k)=0$ :

$$
m_{k, 0}=\sharp\left\{i: u_{i}(k)=v_{i}(k)=0\right\} .
$$

If we take a look at one particular polynomial $P_{u_{i}(k), v_{i}(k)}$ of the above sum, we can find to it a unique predecessor at the step $k-1$, say $P_{u_{i}(k-1), v_{i}(k-1)}$. We can continue and eventually get back to the original $P_{0,0}=P_{u_{i}(0), v_{i}(0)}$. Thus, the points $\left\{i: u_{i}(k)=v_{i}(k)=0\right\}$ can be identified with distinct paths in a two-dimensional lattice, starting and terminating at the origin, composed of edges $e_{1}=(1,0), e_{2}=(-1,1), e_{3}=(0,-1)$, in accordance with the recurrence relations, and never leaving the non-negative quadrant. This is in analogy to the classical Chebyshev polynomials of the second kind and their orthogonalizing measure, where the task of calculating the moments amounts to calculating the distinct paths in the one-dimensional lattice, starting and terminating at the origin, composed of positive and negative unit steps, and never leaving the non-negative semi-axis, 
known as Dyck or Catalan paths. A method of calculating these numbers is to shift the paths upwards by one, thus getting an equivalent requirement on the paths to start and terminate at the point 1 and never to pass through the point 0 . A reflection argument can then be easily applied.

We shall extend the latter observation to the two-dimensional case. Due to the symmetry properties of the problem, the lattice is best presented as a triangular tesselation of the plane. The non-negative quadrant corresponds thus to the region limited by the $x$ and $y$ axes. The edges $e_{1}, e_{2}$ and $e_{3}$ coincide now with suitably oriented walls of the tesselation triangles. We can restate our problem, similarly as in the one-dimensional case, by requiring our paths to start at the point $A=(1,1)$ and never to touch the walls of the non-negative region.

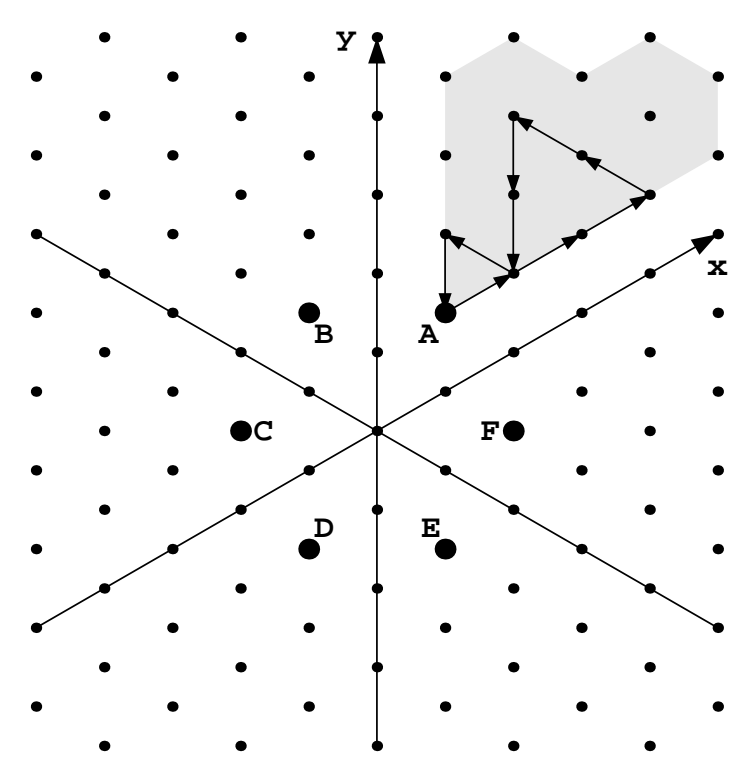

Fig. 1. The lattice with a sample path $\left(e_{1}, e_{1}, e_{1}, e_{2}, e_{2}, e_{3}, e_{3}, e_{2}, e_{3}\right)$

In the sequel we shall need the symbol $d(x, y, n)$ defined as the number of distinct paths from the point $(x, y)$ to the point $A=(1,1)$, composed of exactly $n$ edges and never touching the walls of the positive region. It is straightforward to observe that $d(x, y, n)$ is determined by the following recurrence relations

$$
\begin{aligned}
& d(1,1,0)=1, \\
& d(x, y, 0)=0, \quad x \neq 1 \quad \text { or } \quad y \neq 1, \\
& d(0, y, n)=0 \\
& d(x, 0, n)=0 \\
& d(x, y, n)=d(x+1, y, n-1)+d(x-1, y+1, n-1)+d(x, y-1, n-1),
\end{aligned}
$$

and that $m_{k, 0}=d(1,1, k)$. 
We denote by $\mathcal{R}_{n}(X ; Y)$ the set of distinct paths from the point $X$ to the point $Y$, in exactly $n$ steps, without any restriction on touching the walls and by

$$
\mathrm{R}_{n}(X ; Y)=\sharp \mathcal{R}_{n}(X ; Y)
$$

its cardinality.

Lemma 1. Let $X=(x, y)$ and $Y=(\xi, \eta)$ be any points of the lattice. Let

$$
\left(e_{\iota(1)}, e_{\iota(2)}, \ldots, e_{\iota(n)}\right), \quad \iota(i)=1,2,3
$$

be a path from $\mathcal{R}_{n}(X ; Y)$. Then for every such path the numbers of edges of the three kinds,

$$
k_{1}=\sharp\{i \mid \iota(i)=1\}, k_{2}=\sharp\{i \mid \iota(i)=2\}, k_{3}=\sharp\{i \mid \iota(i)=3\}
$$

are the same and can be determined from $n, X$ and $Y$.

Proof. Any path $\left(e_{\iota(1)} e_{\iota(2)} \ldots e_{\iota(n)}\right)$ starting at $X=(x, y)$ can be seen to terminate at $\left(x+k_{1}-k_{2}, y+k_{2}-k_{3}\right)$. We have thus the following equation system:

$$
\begin{aligned}
k_{1}-k_{2} & =\xi-x, \\
k_{2}-k_{3} & =\eta-y, \\
k_{1}+k_{2}+k_{3} & =n,
\end{aligned}
$$

which gives the solution

$$
\begin{aligned}
& k_{2}=\frac{n+x-\xi+\eta-y}{3}, \\
& k_{1}=k_{2}+\xi-x, \\
& k_{3}=k_{2}+y-\eta .
\end{aligned}
$$

Clearly if $k_{2}$ is not an integer, such paths do not exist.

We are now in a position to present the main theorem.

THEOREM 2. For $x, y \geq 0$

$$
\begin{aligned}
d(x, y, n) & =\mathrm{R}_{n}((x, y) ; A)-\mathrm{R}_{n}((x, y) ; B) \\
& +\mathrm{R}_{n}((x, y) ; C)-\mathrm{R}_{n}((x, y) ; D) \\
& +\mathrm{R}_{n}((x, y) ; E)-\mathrm{R}_{n}((x, y) ; F) .
\end{aligned}
$$

Proof. Let us write $K=\frac{n+x-y}{3}$. Then with the use of Lemma 1 the right hand side can be seen to be:

$$
\begin{aligned}
r h s & =\left(\begin{array}{c}
n \\
K
\end{array}\right)\left(\begin{array}{c}
n-K \\
K-y+1
\end{array}\right)-\left(\begin{array}{c}
n \\
K-1
\end{array}\right)\left(\begin{array}{c}
n-K+1 \\
K-y+1
\end{array}\right)+\left(\begin{array}{c}
n \\
K-1
\end{array}\right)\left(\begin{array}{c}
n-K+1 \\
K-y
\end{array}\right) \\
& -\left(\begin{array}{c}
n \\
K
\end{array}\right)\left(\begin{array}{c}
n-K \\
K-y-1
\end{array}\right)+\left(\begin{array}{c}
n \\
K+1
\end{array}\right)\left(\begin{array}{c}
n-K-1 \\
K-y-1
\end{array}\right)-\left(\begin{array}{c}
n \\
K+1
\end{array}\right)\left(\begin{array}{c}
n-K-1 \\
K-y
\end{array}\right)
\end{aligned}
$$

where $\left(\begin{array}{l}a \\ b\end{array}\right)=0$ for $b<0$ or $b>a$. All the defining recurrence relations for $d(x, y, n)$ defined in equations (3) can be seen to be satisfied by rhs by direct computation. 
Corollary 3. Since $m_{n, 0}=d(1,1, n)$ we get

$$
m_{3 k, 0}=\frac{2(3 k) !}{k !(k+1) !(k+2) !}=\frac{2}{k(k+1)(k+2)}\left(\begin{array}{c}
3 k \\
k
\end{array}\right)\left(\begin{array}{c}
2 k \\
k+1
\end{array}\right),
$$

moreover, $m_{n, 0}=0$ for $n \neq 3 k$.

REMARK 4. We have not been able to find a closed form expression for the general case $m_{k, l}$, and leave it as an open question.

\section{References}

[DX] C. F. Dunkl and Y. Xu, Orthogonal Polynomials of Several Variables, Encyclopedia of Mathematics and its Applications 81, Cambridge University Press, Cambridge, 2001.

[K] T. H. Koornwinder, Orthogonal polynomials in two variables which are eigenfunctions of two algebraically independent partial differential operators, I. Nederl. Akad. Wetensch. Proc. Ser. A 77, Indag. Math. 36 (1974), 48-58. 
